\title{
Study on Lapping Position Control of Spiral Bevel Gear Based on NURBS Surface Fitting
}

\author{
Jianjun Yang ${ }^{1,2, a}$, Linnan Liü,b \\ ${ }^{1}$ School of Mechatronics Engineering, Henan University of Science \& Technology, Luoyang \\ 471003, China \\ ${ }^{2}$ Collaborative Innovation Center of Machinery Equipment Advanced Manufacturing of Henan \\ Province, Luoyang 471003, China \\ ahohooyang@126.com, b412534894@qq.com
}

Keywords: Spiral bevel gear; Lapping; NURBS surface; TCA; Position control

Abstract: The lapping quality and meshing performance of spiral bevel gear depend highly on the exact control over the lapping position on tooth surface. In this paper, the non-uniform rational B-spline (NURBS) surface is used to fit the real measured tooth surface. Based on the tooth contact analysis (TCA) for real measured tooth surface, the mathematic relationship between the adjusting parameters $(V / H / J)$ of lapping movement and meshing position is established. The fixed point lapping and whole tooth surface lapping can be achieved through the lapping point control over position and movement direction.

\section{Introduction}

Spiral bevel gears have been widely used in the fields of aerospace, automotive, instrumentation. At present, TCA is widely used in the design process of spiral bevel gears [1] and the gear lapping technique is applied to reduce the vibration and noise of gear transmission systems [2]. But the existence of machining error may cause the worse lapping quality of gear tooth surfaces [3]. How to achieve the desired lapping effects of spiral bevel gear is particularly significant. Su Zhijian et al. fitted the tooth surface equation with the non-uniform rational B-spline [4]. According to the theoretical tooth surface, Wei Bingyang et al. established the kinematics model of lapping process [5]. However, there are limited reports on the kinematics model of lapping process of the real tooth surface. In view of this, this paper uses the NURBS surface fitting to obtain the digital tooth surface which is highly close to the real tooth surface, and establishes the mathematical relationship between the adjusting parameters $(V / H / J)$ of lapping movement and meshing position. Finally, the simulation of TCA contact pattern is carried out.

\section{Representation of Digital Tooth Surface of Spiral Bevel Gear}

In this paper, JD45+ gear measuring center is used to measure 5 data points along the direction of tooth height $(v)$ and 9 data points along the direction of tooth length $(u)$, as shown in the Figure 1.

According to the reference [6], the expression of the double third order NURBS surface can be written as:

$$
S(u, v)=\frac{\sum_{i=1}^{m} \sum_{j=1}^{n} N_{i, 3}(u) N_{j, 3}(v) w_{i, j} P_{i, j}}{\sum_{i=1}^{m} \sum_{j=1}^{n} N_{i, 3}(u) N_{j, 3}(v) w_{i, j}} .
$$

In which, $m$ is the number of control points along direction $u ; n$ is the number of control points along direction $v ; P_{i j}$ mean the control points of the surface; $w_{i j}$ denote the weight factors of $P_{i j}$; $N_{i, 3}$ and $N_{j, 3}$ are the B-Spline basis functions along the direction $u$ and the direction $v$, respectively.

Take the fitting of the concave surface of the pinion as an example, as shown in the Figure 2. 


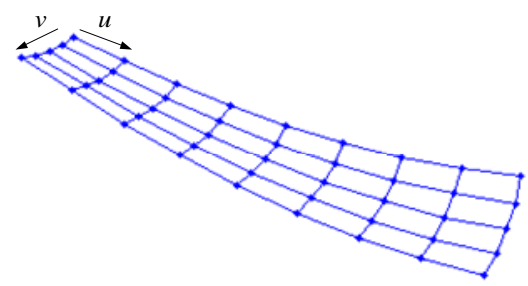

Fig.1 Distribution diagram of measuring points

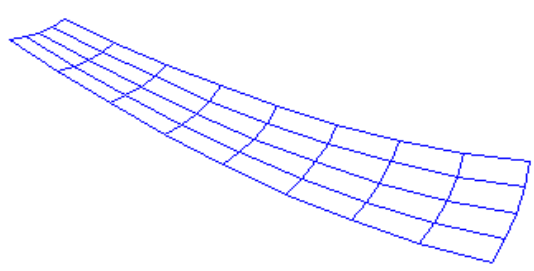

Fig.2 NURBS surface

After measuring the coordinates of data points of the real tooth surface, the double third order NURBS surface approximated to the real tooth surface is obtained by interpolation and inverse algorithm, which is shown as follows:

$$
\mathbf{r}_{i}=\mathbf{r}_{i}\left(u_{i}, v_{i}\right), \quad i=1,2 .
$$

Here: 1 and 2 represent the pinion and the gear, respectively. $u$ and $v$ are surface parameters.

In order to verify the precision of digital tooth surface, $5 \times 9$ points on the theoretical tooth surface of spiral bevel gear are extracted and fitted to generate the digital tooth surface. In the same range, $15 \times 27$ points are selected to calculate the normal distance between the theoretical tooth surface and the digital tooth surface, which can be used as the fitting errors. The maximum fitting error curves of each column are shown in the Figure 3. Because of the large curvature of the pinion tooth surface, the fitting accuracy is lower than that of the gear, but the maximum fitting error is not more than $0.1 \mathrm{um}$, which can meet with the precision requirements.

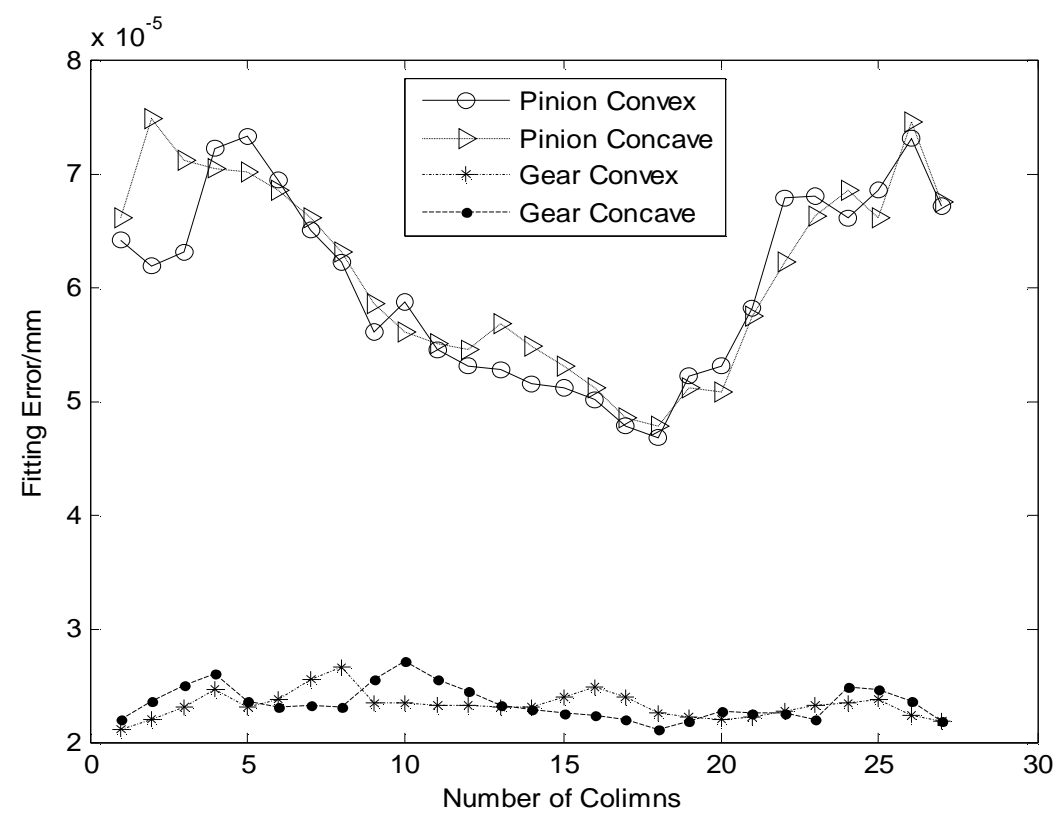

Fig.3 Curve of fitting error

\section{Kinematics Model of Lapping Process on Digital Tooth Surface}

Figure 4 is the coordinate relation of tooth surface meshing. $S_{1}\left(O_{1}-X_{1} Y_{1} Z_{1}\right)$ and $S_{2}\left(O_{2}-X_{2} Y_{2} Z_{2}\right)$ are the coordinate system with the pinion and the gear, respectively. $S_{h}\left(O_{h}-X_{h} Y_{h} Z_{h}\right)$ is a space fixed coordinate system, which connect rigidly with the bed of the machine tool.

The position vector and the unit normal vector of the meshing point on the tooth surface are expressed as equation (3). Among it, $\varphi$ is the angle of tooth meshing.

$$
\left\{\begin{array}{c}
\mathbf{r}_{h 1}\left(\mathrm{u}_{1}, v_{1} ; \varphi_{1}, V, H, J\right)=\mathbf{r}_{h 2}\left(u_{2}, v_{2} ; \varphi_{2}\right) \\
\mathbf{n}_{h 1}\left(\mathrm{u}_{1}, v_{1} ; \varphi_{1}\right)=\mathbf{n}_{h 2}\left(u_{2}, v_{2} ; \varphi_{2}\right)
\end{array} .\right.
$$

In addition, the meshing equation at the nominal contact point should be satisfied as: 


$$
\mathbf{n}_{h}^{(2)} \cdot \mathbf{v}_{h}^{(12)}=f\left(u_{1}, v_{1}, \varphi_{1}, u_{2}, v_{2}, \varphi_{2}, V, H, J\right)=0 .
$$

Here, $\mathbf{n}_{h}^{(2)}$ means the normal vector of the meshing point, $\mathbf{v}_{h}^{(12)}$ is the relative velocity of the meshing point.

In the process of gear lapping, the equal clearance of two tooth should be ensured, so the parameters $(J / H)$ should satisfy the following formula:

$$
J+H \tan \delta_{1}=0 .
$$

Here, $\delta_{1}$ is the pitch cone angle of the pinion.

Figure 5 shows the projection of the gear (convex) in a axial section, $O_{2}$ is the pitch cone vertex of the gear, $P$ is a node, $A$ is the distance of the pitch cone, $M$ is the nominal contact point, $M^{*}$ is the center point of the lapping area. At the same time, the change of the nominal contact point in tooth length and tooth height is $s_{1}$ and $s_{2}$ (the arrow in the figure 5 shows positive direction). The coordinates $\left(X_{2}^{*}, Y_{2}^{*}\right)$ of the point $\left(M^{*}\right)$ and the corresponding points of the three-dimensional coordinate system of the tooth surface meet the following relationship:

$$
\left\{\begin{array}{c}
\mathrm{x}_{2}=X_{2}^{*} \\
\sqrt{\mathrm{y}_{2}^{2}+\mathrm{z}_{2}^{2}}=Y_{2}^{*}
\end{array} .\right.
$$

Where, $\left(x_{2}, y_{2}, z_{2}\right)$ is the coordinate component of the tooth surface equation.

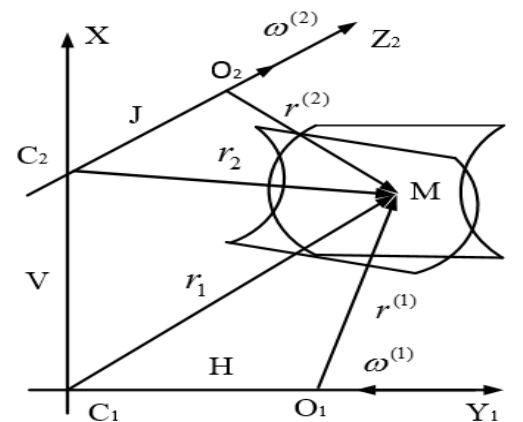

Fig. 4 Relative position of two tooth surfaces

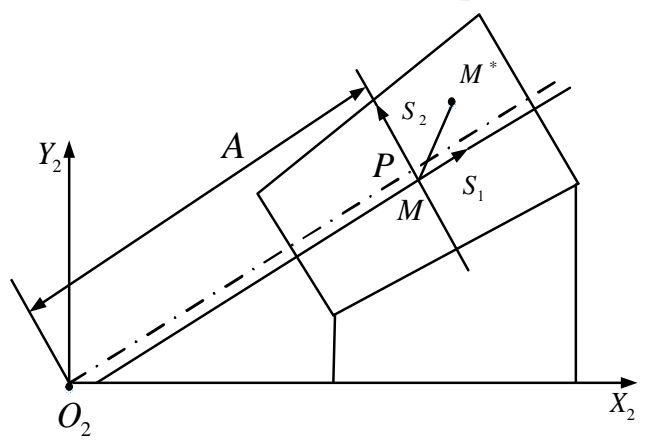

Fig. 5 Projection coordinate of the gear tooth surface

According to equation (6), $u_{2}$ and $v_{2}$ can be calculated. So adjusting parameters $(V / H / J)$ can be solved based on equations $(3) \sim(5)$.

\section{Tooth Contact Analysis}

A pair of spiral bevel gears as an example. The parameters are shown in Table 1.

Table 1 Basic parameters of gear pair

\begin{tabular}{lccc}
\hline Parameters & Pinion & & Gear \\
\hline The number of teeth & 8 & & 31 \\
Shaft angle(deg) & & 90 & \\
Moudule(mm)Error! No & & 5.53 & \\
bookmark name given. & & 20 & \\
$\begin{array}{l}\text { Pressure angle(deg) } \\
\text { Spiral angle(deg) }\end{array}$ & & 35 & \\
Hand of spiral & Left & & Right \\
Face width(mm) & & 26.7 & \\
\hline
\end{tabular}

In the theoretical installation position, the values of $V, H, J$ are 0 , solving equations (3) (4) and using the method of TCA, the contact trace and contact pattern of the gear convex surface can be obtained, as shown in Figure 6a. TCA results are shown in Figure $6 b \sim 6$ e. The equations (3) (6) can 
be used to calculate the corresponding adjustment values $(V / H / J)$, as shown in the Table 2 .

If $s_{1}=0.2 b, s_{2}=0$ and $s_{1}=-0.2 b, s_{2}=0$ ( $b$ is the gear tooth length) are taken separately, then the gear tooth contact pattern will move $b / 5$ to the big end and the small end ,respectively. If $s_{1}=0.2 b, s_{2}=0.2 h$ ( $h$ is the gear tooth height) and $s_{1}=-0.2 b, s_{2}=-0.2 h$ are taken separately, then the tooth contact pattern will be move $b / 5$ to the big end and the small end, and move $h / 5$ to the tooth top and the tooth root, respectively. The relationship model of adjusting parameters $(V / H / J)$ and the contact position of the tooth surface is established based on the equations (3) (6).

Table 2 Relationship between the values (V/H/J) and the contact position of tooth surface

\begin{tabular}{c|c|c|c|c}
\hline \multicolumn{2}{c|}{ Projection coordinates } & \multicolumn{3}{|c}{ Positive rotation surface } \\
\hline$s_{1}$ & $s_{2}$ & $V$ & $H$ & $J$ \\
\hline$-0.2 \mathrm{~b}$ & 0 & 0.71801 & -0.58154 & 0.15007 \\
$0.2 \mathrm{~b}$ & 0 & -0.77627 & 0.57734 & -0.16401 \\
$0.2 \mathrm{~b}$ & $0.2 \mathrm{~h}$ & -0.69123 & 0.53835 & -0.16573 \\
$-0.2 \mathrm{~h}$ & $-0.2 \mathrm{~h}$ & 0.68058 & -0.53146 & 0.15609 \\
\hline
\end{tabular}
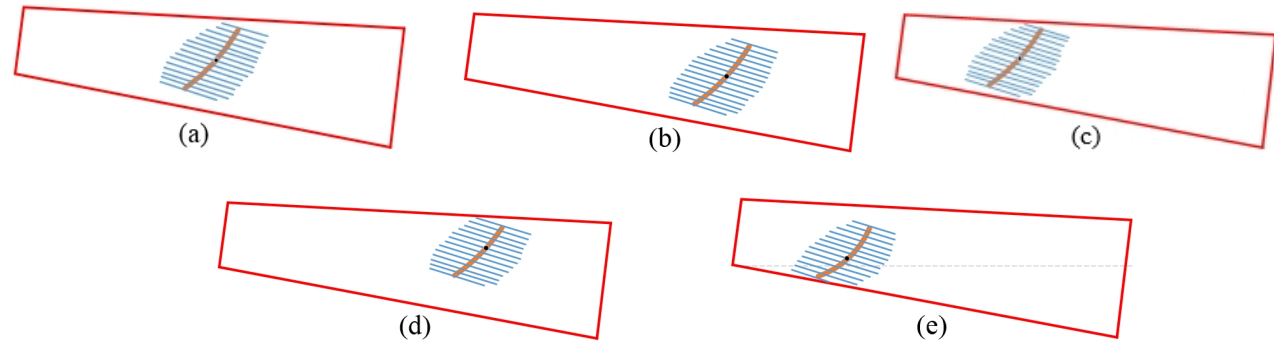

Fig. 6 Simulation of convex contact pattern of gear

\section{Conclusions}

In this paper, a method of lapping movement analysis with high precision digital tooth surface is presented. The mathematical model of the relationship between the $V / H / J$ coordinate and the tooth surface meshing is established. TCA method is used to simulate the contact path and the contact pattern of the tooth surface. The simulation results show that the mathematical model can accurately control the position and direction of the contact point of the lapping tooth surface. The establishment of the model provides theoretical guidance for the accurate lapping of spiral bevel gears.

\section{Acknowledgements}

Financial assistance was provided by the National Natural Science Foundation of China (No. 51375144) and the Program for Science \& Technology Innovation Talents in Universities of Henan Province (No.15HASTIT025).

\section{References}

[1] Deng Xiaozhong, Fang Zongde, et al. Journal of aerospace power, 2003, 18 (6): 744-748.

[2] Zeng Jiancheng, Zeng Tao, et al. Microcomputer information, 2010, 26 (25): 157-158.

[3] Xiao Chunfang. Chemical Industry Press, 2015, 39-40.

[4] Su Zhijian, Wu Xutang, et al. Journal of Xi'an Jiaotong University, 2005, 39 (1): 17-20.

[5] Wei Bingyang, Zhou Yanwei, et al. Modern manufacturing engineering, 2005 (4): 17-19.

[6] Les Piegl. Tsinghua University Press, 2010, 86-93. 